\title{
Chemical Abundances of Metal-poor stars in Dwarf Galaxies
}

\author{
Kim A. Venn ${ }^{1}$, Pascale Jablonka ${ }^{2}$, Vanessa Hill ${ }^{3}$, Else Starkenburg ${ }^{4}$, \\ Bertrand Lemasle $^{5}$, Matthew Shetrone ${ }^{6}$, Mike Irwin ${ }^{7}$, John Norris ${ }^{8}$, \\ David Yong $^{8}$, Gerry Gilmore ${ }^{7}$, Stephania Salvadori ${ }^{9}$, Asa Skuladottir ${ }^{9}$ \\ and Eline Tolstoy ${ }^{9}$ \\ ${ }^{1}$ Dept. of Physics \& Astronomy, University of Victoria, Victoria, BC, V8P 5C2, Canada \\ email: kvenn@uvic.ca \\ ${ }^{2}$ Laboratoire d'astrophysique, EPFL, Observatoire de Sauverny, 1290 Versoix, Switzerland \\ ${ }^{3}$ Obs. de la Cote d'Azur, 06304 Nice Cedex 4, France \\ ${ }^{4}$ Leibniz-Institute fur Astrophysik Potsdam, 14482 Potsdam, Germany \\ ${ }^{5}$ Anton Pannekoek Inst. for Astronomy, Univ. of Amsterdam, 1090 GE Amsterdam, \\ Netherlands \\ ${ }^{6}$ McDonald Observatory, Univ. of Texas at Austin, Austin TX 78712, USA \\ ${ }^{7}$ Institute of Astronomy, Univ. of Cambridge, Madingley Road, Cambridge, CB3 0HA, UK \\ ${ }^{8}$ RSAA, Australian National Univ., Mount Stromlo Observatory, Weston ACT 2611, Australia \\ ${ }^{9}$ Kapteyn Astronomical Inst., Univ. of Groningen, 9747 AD Groningen, Netherlands
}

\begin{abstract}
Stars in low-mass dwarf galaxies show a larger range in their chemical properties than those in the Milky Way halo. The slower star formation efficiency make dwarf galaxies ideal systems for testing nucleosynthetic yields. Not only are alpha-poor stars found at lower metallicities, and a higher fraction of carbon-enhanced stars, but we are also finding stars in dwarf galaxies that appear to be iron-rich. These are compared with yields from a variety of supernova predictions.
\end{abstract}

Keywords. stars: abundances, galaxies: dwarf, evolution, Local Group, stellar content.

\section{Introduction}

It has been known for more than a decade now that "the overwhelming majority of Milky Way stars, those in the Galactic thick disk and thin disk, seem to have nothing at all to do with dwarf galaxy origins" (Gilmore 2012). As summarized by Tolstoy et al. (2009), the ratio of alpha-elements to iron are lower in the stars in dwarf galaxies for metallicities above $[\mathrm{Fe} / \mathrm{H}] \sim-2$. This has been found for stars in the Sculptor, Carina, Fornax, and LMC dwarf galaxies, and the Sagittarius dwarf galaxy remnant. The early results have been confirmed by larger data samples (e.g., Lemasle et al. 2012, 2014, Hendricks et al. 2014), and extended to lower mass dwarfs, such as Bootes I and Segue I (e.g., Feltzing et al. 2009, Norris et al. 2010, Frebel et al. 2014).

The picture is less clear when comparing the chemistry of the stars in the Galactic halo with those in dwarf galaxies with metallicities $[\mathrm{Fe} / \mathrm{H}]<-2.0$. An examination of the stellar abundances of $[\mathrm{Ca} / \mathrm{Fe}]$ and $[\mathrm{Mg} / \mathrm{Fe}]$ in stars below metallicity $[\mathrm{Fe} / \mathrm{H}]=-2$ is shown in Fig. 1. The uncertainties in each individual stars are typically $<0.2$ dex. While the general trend of high [alpha/Fe] ratios (above the solar ratio) appears in all systems, the dispersion is larger in the dwarf galaxies, and includes several stars with very low $[$ alpha/Fe] abundances (below the solar ratio). 

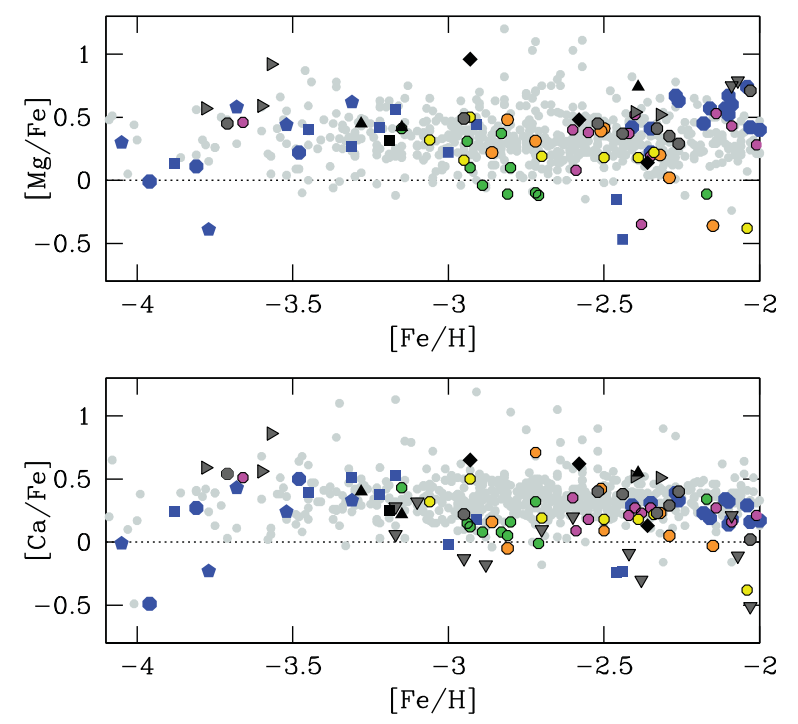

Figure 1. $[\mathrm{Mg} / \mathrm{Fe}]$ and $[\mathrm{Ca} / \mathrm{Fe}]$ for stars in the Milky Way (grey) and dwarf galaxies. These include abundances summarized by Frebel 2010, and additional new references for Sculptor (blue; Jablonka et al. 2015, Simon et al. 2015, Starkenburg et al. 2013, Tafelmeyer et al. 2010), Sextans (green; Tafelmeyer et al. 2010) Carina (orange; Norris et al. 2016, Venn et al. 2012, Lemasle et al. 2012) Draco and UMi (yellow), and the ultra faint dwarf galaxies (black; Frebel 2014 for Segue 1 and Aden et al. 2011 for Hercules).

\section{Element Abundances in Metal-Poor Stars}

At the lowest metallicities, the detailed chemical abundances have been modelled as enrichment by a variety of core collapse SN, including a range of progenitor masses, $\mathrm{SN}$ explosion energies, and mixing and fallback prescriptions. Iwamoto et al. (2005) have found that "faint supernovae" with extensive mixing and fallback during the SN II explosion produce decreasing yields with increasing atomic number (mitigated by the odd-even effect); and that these yields reproduce the element distribution for the ultra metal-poor stars HE1327-2326 and HE0107-5240 better than predictions from the metalfree massive models by Heger \& Woosley $(2002,2008)$. Neither of these models produce the heavy neutron capture elements.

The core collapse supernova models by Wanajo (2013) can reproduce the solar-system r-process distribution by tuning the initial core masses. These models are also able to reproduce the heavy element distribution in metal-poor Galactic stars, such as CS 31082001. However, Wanajo et al. (2014) have also been able to make these same heavy element distributions with a variety of compact binary mergers. This latter scenario is an interesting alternative for the site of the r-process (also see Shen et al. 2015), though rely on timescale arguments for making and merging two neutron stars.

In Fig. 2, the $[\mathrm{Ba} / \mathrm{Fe}]$ ratios for metal-poor stars in the Galaxy and dwarf galaxies are shown. The general distribution and dispersions are quite similar for the majority of stars, implying the sites and yields for the r-process are similar in these systems. Again, the uncertainties in the abundances ratios are estimated as $<0.2$ dex. Only the upper limits on $[\mathrm{Ba} / \mathrm{Fe}]$ for stars in the dwarf galaxies show an unexpected result, as highlighted by Frebel et al. (2014). The intermediate metallicity stars (with $[\mathrm{Fe} / \mathrm{H}]>-2.5$ ) in the ultra faint dwarf galaxies, Segue 1, Com Ber, and Hercules, have extremely low [Ba/Fe] values or upper limits. This suggests these stars formed from gas that was not enriched in r-process elements, at all, yet they have high iron abundances. This is not seen in the 


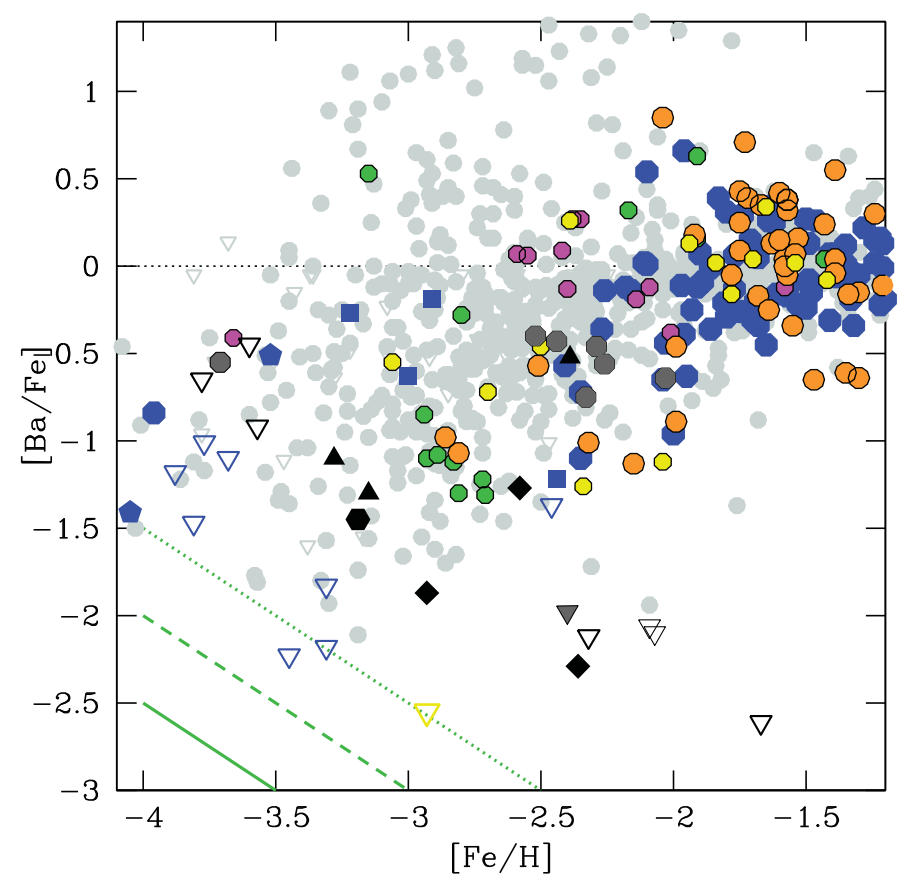

Figure 2. $[\mathrm{Ba} / \mathrm{Fe}]$ for stars in the Milky Way (grey) and dwarf galaxies (colours as in 1). Upper limits for stars in dwarf galaxies (only) are noted as open downward-pointing open triangles. Detection limits for the Ba II $4554 \mathrm{~A}$ spectral line are shown as green lines (10 mA as dotted, $3 \mathrm{~mA}$ as dashed, $1 \mathrm{~mA}$ as solid), for a red giant with $\mathrm{T}=4500 \mathrm{~K}$, following Roederer (2013).

Galaxy nor the classical dwarf galaxies. This observation has been interpreted by Frebel \& Norris (2015) as the result of a single (or few) high-mass metal-free supernova event that also removed the gas in these lowest mass galaxies.

\section{Iron-rich Stars in Dwarf Galaxies}

At intermediate metallicities, $[\mathrm{Fe} / \mathrm{H}]>-2.5$, another phenomenon is found in dwarf galaxies, where some stars appear to be enriched in iron-group elements. This is seen as an unusually low $[\mathrm{X} / \mathrm{Fe}]$ ratios for nearly all elements, except those in the iron-group. One example is Star 612 in the Carina dwarf galaxy (Venn et al. 2012), though others have now been found in Carina (Norris et al. 2016) and Sculptor (Jablonka et al. 2015). Similar stars have been found in the (outer) halo of the Galaxy by Ivans et al. (2003) and Jacobson et al. (2015).

One possibility is that the iron-enrichment comes from inhomogeneous mixing of the interstellar medium at later times. Pockets of SN Ia material may occur if the gas is poorly mixed. Some chemical evolution models (e.g., Revaz \& Jablonka 2012, Wise et al. 2012, Romano et al. 2015) support inhomogeneous mixing on sufficiently long timescales only in the lower mass dwarf galaxies.

Another option has been suggested by Kobayashi et al. (2015) where SN Iax have a more significant role in the chemical evolution of a dwarf galaxy. SN Iax are those from a higher mass (hybrid, $\mathrm{C}+\mathrm{O}+\mathrm{Ne}$ ) white dwarf, formed at lower metallicities (even as low as $[\mathrm{Fe} / \mathrm{H}]=-2.5)$. 


\section{Carbon-rich Stars in Dwarf Galaxies}

Carbon-enhancements $([\mathrm{C} / \mathrm{Fe}]>0.7)$ are commonly found in metal-poor stars in the Galaxy and typically associated with binary systems (e.g., Starkenburg et al. 2014). However, the CEMP-no stars may be a different class of these stars, formed from primordial faint supernovae and dominating the early metal enrichment in dwarf galaxies. While CEMP-no stars are found in the Galaxy and the ultra faint dwarf galaxies (e.g., Beers \& Christlieb 2005, Norris et al. 2013), they have only recently been found also in the classical dwarf galaxies (Skuladottir et al. 2015). Initial interpretations were that the early chemical evolution of the classical dwarf galaxies may have differed from those of the ultra faint dwarfs, however a recent analysis of cosmological models of dwarf galaxies has shown that CEMP-no stars would have formed in all systems, and with equal fractions in the populations with $[\mathrm{Fe} / \mathrm{H}]<-4$ (Salvadori et al. 2015). The difficulty is in finding these stars once the peak in the metallicity distribution function moves from $[\mathrm{Fe} / \mathrm{H}]=$ -3 to -2 , as in the higher mass classical dwarf galaxies. Also the classical dwarf galaxies are further away, meaning that only the brightest stars can be observed. Comparisons of the metallicities of the stars from CaT surveys versus those from high resolution studies shows that more metal-poor stars are detected at lower magnitudes (e.g., Lemasle et al. 2012).

\section{Conclusions}

The element abundance ratios in the most metal-poor stars $([\mathrm{Fe} / \mathrm{H}]<-3)$ in dwarf galaxies are excellent tests for the variety of SN II yields and other supernova models. Most elements show larger dispersions in $[\mathrm{X} / \mathrm{Fe}]$ in dwarf galaxies, than similar stars in the Galactic halo. Spectral lines of some of the more interested elements (such as barium) can be so weak that we are near the detection limits of our high resolution spectrographs, even on the 8-10 meter telescopes. Also, the CEMP stars are likely in all dwarf galaxies, though can be harder to find in the more distant and higher mass (higher mean metallicity) classical dwarf galaxies.

The intermediate-metallicity stars $([\mathrm{Fe} / \mathrm{H}] \sim-2)$ can show the largest deviations from similar metallicity stars in the Galactic halo, due to differences in the later chemical evolution stages of the dwarf galaxies. This can include effects due to inhomogeneous mixing of the interstellar medium, including SN Ia pockets of iron-enrichment, in the lower mass dwarf galaxies.

\section{References}

Aden, D. et al. 2011, A\&A A, 525, 153

Feltzing, S. et al. 2009, $A \mathscr{E} A$, 508, 1

Frebel, A. 2010, in Astron. Nachr. 331, 474

Frebel, A., Simon, J. D., Kirby, E. N. 2014, ApJ, 786, 74

Frebel, A., Norris, J. E. 2015, ARAA, 53, 631

Gilmore, G. 2012, in Assembling the Puzzle of the Milky Way, Le Grand-Bournand, France.

Heger, A., Woosley, S. E. 2003, ApJ, 567, 532

Heger, A., Woosley, S. E. 2010, ApJ, 724, 341

Hendricks, B. et al. 2014, ApJ, 785, 102

Ivans I., et al. 2003, ApJ, 592, 906

Iwamoto, N., et al. 2005, Sci, 309, 451

Jablonka, P., et al. 2015, A\& A, 583, 67

Jablonka, P., et al. 2016, in prep

Kobayashi, C., et al. 2015, ApJ, 804, 24 
Lemasle, B., et al. 2012, A\&A, 538, 100

Lemasle, B., et al. 2014, $A \mathscr{E} A, 572,88$

Norris, J. E., et al. 2010, ApJ, 723, 1632

Norris, J. E., et al. 2013, ApJ, 762, 28

Norris, J. E., et al. 2016, in prep

Revaz, Y., Jablonka, P 2012, A\& A, 538, 82

Roederer, I.U. 2013 AJ, 145, 26

Romano, D., Bellazzini, M., Starkenburg, E., Leaman, R. 2015, MNRAS, 446, 4220

Salvadori, S., Skuladottir, A., Tolstoy, E. 2015 MNRAS, 454, 1320

Shen, S., Cooke, R. J., Ramirez-Ruiz, E., et al. 2015, ApJ, 807, 115

Simon, J. D. et al. 2015, ApJ, 802, 93

Skuladottir, A., et al. 2015, A\&A $, 574,129$

Starkenburg, E., et al. 2014, MNRAS, 441, 1217

Starkenburg, E., et al. 2013, MNRAS, 429, 725

Tafelmeyer, M., et al. 2010, A\&A, 524, A58

Tolstoy, E., et al. 2009, ARAA, 47, 371

Venn, K. A., et al. 2012, ApJ, 751, 102

Wanajo, S. 2013, ApJ, 770, 22

Wanajo, S. et al. 2014, ApJ, 789, 39

Wise, J. et al. 2012, ApJ, 745, 50 